\title{
Number of Electrooculogram Electrodes
}

National Cancer Institute

\section{Source}

National Cancer Institute. Number of Electrooculogram Electrodes. NCI Thesaurus.

Code C154907.

The number of electrooculogram electrodes used in the assessment. 